\title{
Biomarkers of Oxidative Stress for Human Health: Experiment of Different Swimming Exercise on Male Wistar Rats
}

\author{
Agus Hariyanto ${ }^{1}$, Anindya Mar'atus Sholikhah ${ }^{2}$, Yetty Septiani Mustar ${ }^{3}$ \\ \{agushariyanto@unesa.ac.id\} \\ Faculty of Sport Science, Universitas Negeri Surabaya, East Java, Indonesia
}

\begin{abstract}
This study aims to compare the biomarker of oxidative stress in rats after subjected to exercise in different intensity. It was an experimental study involving 15 male Wistar rats. Rats were allocated to control (C), moderate swimming training (M) and exhaustive swimming training (EX). Rats in $\mathrm{M}$ group were forced to swim twice a week for 15 minutes, while rats in EX were forced to swim with external load added (5\% of bodyweight) until exhausted or once rats stayed submerge for $10 \mathrm{~s}$. After two weeks of exercise, rats were euthanized and $4 \mathrm{~mL}$ of blood samples were drawn to measure MDA and SOD. The results of this study revealed a significant difference in MDA and SOD level between groups. Rats in EX were found to have highest level of MDA (38.10 \pm $2.29)$ as well as lowest in SOD level $(1.06 \pm 0.06)$ than the peers in $M$. The findings indicate that moderate exercise is the most suitable exercise to prevent oxidative stress.
\end{abstract}

Keywords: exercise, free radical, intensity, malondialdehyde, oxidative stress, superoxide dismutase.

\section{Introduction}

Physical activity that is carried out regularly as part of a healthy lifestyle will have a positive impact on health [1]. Studies reported that physical activity can lower the risk of several degenerative diseases, such as cardiovascular disease [2][3], cancer [4], and diabetes mellitus type 2, as well as reduce osteoporosis [5]. Moreover, adequate physical activity is found to be a strong gene modulator that induce structural changes in the human brain, affecting cognitive function [6]. The benefit of regular exercise is also found to associate with psychological well-being which in turn affect quality of life [7].

While regular exercise or physical activity contributes to numerous benefits on human health system, studies observed the differences in positive effects when the volume and intensity are taken into account 7 . The favourable effects of exercise are lost following the exhaustion [8]. In other words, excessive exercise which depletes the body's adaptive system, or the ability to give positive feedback to training stimulus, can be damaging to human's health [9]. Exercise also represents a physical stress that shatters homeostasis state [10], with working skeletal muscle is the most afflicted organ during physical activity [11]. Several studies stated that exhaustive exercise especially when performed sporadic, can alter inflammatory reactions or structural damage within the muscles, likely due to the enhance production of radical oxygen species (ROS) [8]. When cell is under exhaustive condition, the utilization of oxygen increases 10-15 times and free radicals may be generated beyond the 
body's natural antioxidant defense [12], which is characterized by the changes in oxidative stress biomarkers.

ROS in sufficient level actually plays important role in modulation of numerous cellular function such as signalling process, tissue homeostasis, as well as involving in biological system [13][14]. But when it is overproduced as the result of exhaustive exercise, the damage is inevitable as it can lead to oxidative stress which further causes lipid peroxidation in cells and tissues[15]. Overproduction of ROS also involves in disturbance of several biological processes, including physiological, psychological, and immunological systems[16]. Furthermore, it poses serious threat to antioxidant defence system which later can increase cells susceptibility to oxidative damage [8].

It is well established that exercise is known to induce the generation of free radical. Previous studies have demonstrated that acute aerobic and anaerobic exercise, both maximal or submaximal, can generate ROS [17]. While the exercise-induced oxidative stress has received wide attention, the relationship between exercise and its subcomponent such as intensity and the resultant production of ROS still remains unclear. And the important question about the role of various types of exercise and load or intensity is still an issue under debate. Little is known about which type of exercise or what level of intensity could give more benefit to prevent oxidative stress. Hence, the present study aims to compare the biomarker of oxidative stress in rats after subjected to exercise in different intensity.

\section{Method}

It was an experimental design using post-test only control group design. In this study, five weeks old fifteen male Wistar rats (Rattus norvegicus) weighing 150-200 grams were used. All rats were placed in standard cages and maintained to adapt under environmentally controlled conditions during the study $(25 \pm 1 \mathrm{oC}$ temperature; $50 \pm 1 \%$ relative humidity; and 12:12 hours light-dark cycle) and were provided standard rat chow and tap water ad libitum during experimental period. Afterward, they were divided into three weight-matched groups $(\mathrm{n}=5 / \mathrm{cage})$ : (1) control group (C); (2) moderate intensity swimming training $(\mathrm{M})$, and (4) exhaustive swimming training (EX).

All rats were adapted to the water for 1 week during the acclimatization period. The adaptation was done by putting the animals in shallow water for 1 minutes every day to prevent stress. After a week of acclimatization, the rats were assigned to respective swimming training. The swimming session was done individually in a glasses tank filled up with water that was set on normal temperature and sufficient enough for rat to swim simultaneously. Moderate swimming training was performed every morning at 9 am, two times a week, 15 minutes/day, for two weeks. While exhaustive swimming training was done using the same protocol as moderate exercise but until rats got exhausted. Exhaustion was defined once rat remained submerged in the water for 10 seconds. An external load weighed $5 \%$ of rat's bodyweight was added to EX group to augment the exercise intensity.

After each swimming session, rats were hair-dried to prevent hypothermia stress. 24-h after the end of last swimming session, all rats were euthanized. $5 \mathrm{~mL}$ of intracardiac bloods samples were drawn to measure malondialdehyde (MDA) level and superoxide dismutase (SOD). MDA was measured using thiobarbituric acid reactive substances method (TBARS) and the result was stated as $\mu \mathrm{M}$, while SOD measurement was performed by xanthine oxidase method and the result was stated as $\mathrm{U} / \mathrm{mL}$. 
Data were analysed using standard statistical software package SPSS and presented as mean values and standard deviation (SD). Kolmogorov-Smirnov was used to evaluate the data distribution. The normally distributed data were analysed using Pearson correlation to assess the relationship between MDA and SOD level. Further analysis was carried out by one-way Anova to compare MDA and SOD level between groups, and post-hoc analysis was carried out using LSD test to find out which pair showed significant different. P-value less than 0.05 was considered statistically significant.

\section{Result}

This present study investigated the effect of different intensity of swimming training on oxidative stress biomarker in rats. Rats in all groups were healthy and did not show any significant changes on physical figure and behaviour after a week of acclimatization phase. All rats completed the whole protocol from first week until the end of experimental period.

Table 1. Normality test of oxidative stress biomarker

\begin{tabular}{lccc}
\hline \multicolumn{1}{c}{ Biomarker } & Mean & SD $( \pm)$ & p-value \\
\hline Malondialdehyde $(\mu \mathrm{M})$ & 34.46 & 4.03 & 0.846 \\
Superoxide dismutase $(\mathrm{U} / \mathrm{mL})$ & 1.16 & 0.09 & 0.631 \\
\hline
\end{tabular}

Table 1 shows an overview of normality test on oxidative stress biomarker. The result of Kolmogorov-Smirnov test shows that malondialdehyde (MDA) and superoxide dismutase (SOD) are normally distributed with p-value 0.846 and 0.631 , respectively. The overall mean level of MDA is $34.46 \pm 4.03 \mu \mathrm{M}$, while $\mathrm{SOD}$ is $1.16 \pm 0.09 \mathrm{U} / \mathrm{mL}$ (Table 1 ).

a. The Mean Level of MDA $(\mu \mathrm{M})$

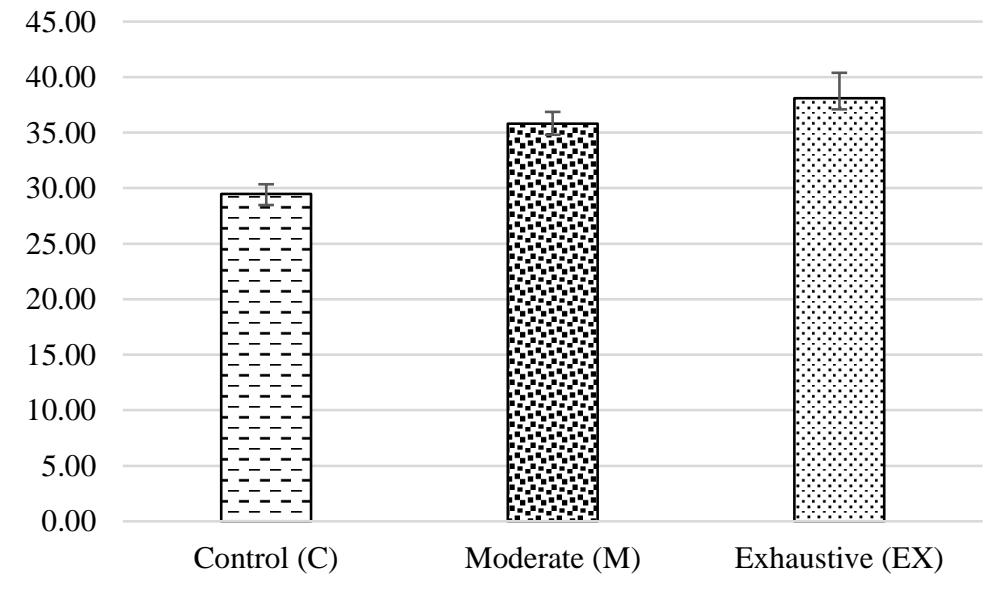


b. The Mean Level of SOD (U/mL)

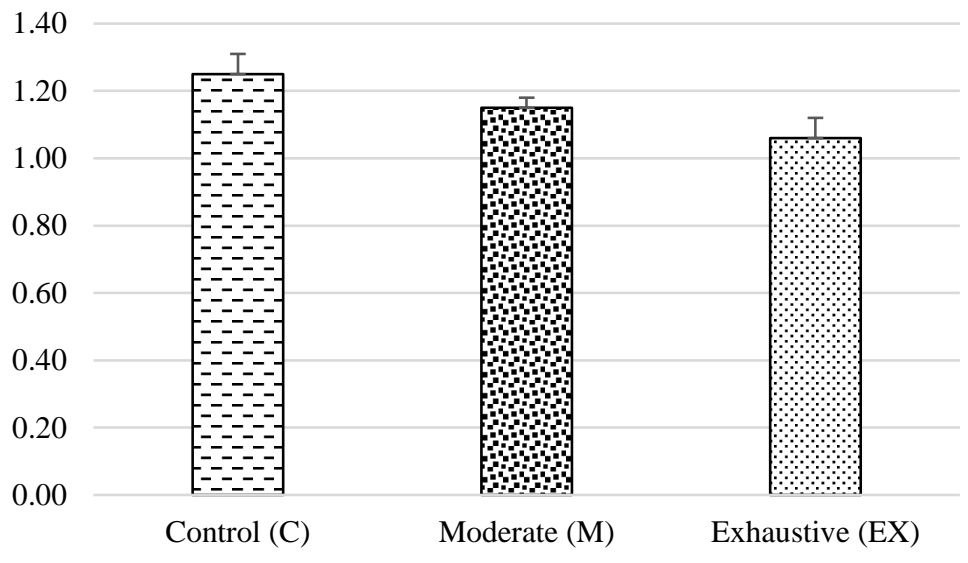

Fig 1. The Mean Level of MDA (a) and SOD (b) in Each Group

The highest level of MDA is $38.10 \pm 2.29 \mu \mathrm{M}$ and it is found in EX group which is assigned to exhaustive exercise. The contrast finding is found in SOD level, where EX group happens to have lowest endogenous antioxidant (SOD) with $1.06 \pm 0.06 \mathrm{U} / \mathrm{mL}$ compared to moderate (M) and control group (C) (Fig. 1).

Table 2. The correlation between Malondialdehyde (MDA) and Superoxide Dismutase (SOD) level

\begin{tabular}{|c|c|c|c|c|c|}
\hline Variable & Group & Mean & SD $( \pm)$ & $\mathbf{r}$ & p-value \\
\hline Malondialdehyde $(\mu \mathrm{M})$ & Control (C) & 29.48 & 0.87 & \multirow{6}{*}{-0.726} & \multirow{6}{*}{0.021} \\
\hline & Moderate (M) & 35.81 & 1.06 & & \\
\hline & Exhaustive (EX) & 38.10 & 2.29 & & \\
\hline \multirow{3}{*}{$\begin{array}{l}\text { Superoxide dismutase } \\
(\mathrm{U} / \mathrm{mL})\end{array}$} & Control (C) & 1.25 & 0.06 & & \\
\hline & Moderate (M) & 1.15 & 0.03 & & \\
\hline & Exhaustive (EX) & 1.06 & 0.06 & & \\
\hline
\end{tabular}

Pearson correlation found a negative correlation between MDA and SOD level with $r=-$ 0.726 . It could be seen that swimming training that were assigned to rats altered the redox balance in favour of oxidative stress, where the increase of MDA level would be followed by the decrease amount of SOD.

Table 3. The comparison of Malondialdehyde (MDA) and Superoxide Dismutase (SOD) level between groups

\begin{tabular}{llll}
\hline \multicolumn{1}{c}{ Variable } & & Group & p-value \\
\hline $\begin{array}{l}\text { Malondialdehyde } \\
(\mu \mathrm{M})\end{array}$ & Control (C) & Moderate $(\mathrm{M})$ & 0.000 \\
& & Exhaustive $(\mathrm{EX})$ & 0.000 \\
\cline { 2 - 4 } & Moderate $(\mathrm{M})$ & Control $(\mathrm{C})$ & 0.000 \\
& & Exhaustive (EX) & 0.036 \\
\cline { 2 - 4 } & Exhaustive (EX) & Control (C) & 0.000 \\
& & Moderate (M) & 0.036 \\
\hline Superoxide dismutase & Control (C) & Moderate (M) & 0.012 \\
$(\mathrm{U} / \mathrm{mL})$ & & Exhaustive (EX) & 0.000 \\
\cline { 2 - 4 } & Moderate (M) & Control (C) & 0.012 \\
\hline
\end{tabular}




\begin{tabular}{llll}
\hline & & Exhaustive (EX) & 0.017 \\
\cline { 2 - 4 } & Exhaustive (EX) & Control (C) & 0.000 \\
& Moderate (M) & 0.017 \\
\hline
\end{tabular}

According to the result of one-way Anova, MDA level in control group (C) shows significant different with those in moderate $(\mathrm{M})$ and exhaustive swimming training group $(\mathrm{EX})$, with $\mathrm{p}=0.000$ for both groups. MDA level in moderate group also differs significantly with the peers in exhaustive exercise $(p=0.036)$. The present study also found significant differences in the level of SOD between control group and moderate $(p=0.012)$ and exhaustive $(\mathrm{p}=0.000)$ groups. Furthermore, rats in moderate group shows significant different with those assigned to exhaustive exercise $(0=0.017)$.

\section{Discussion}

In recent years of clinical research, MDA and SOD have often been used as popular matching indicators of oxidative stress. MDA is one of the aldehyde compounds occurring as a result of lipid peroxidation [18]. MDA reflected the degree of damage in cells caused by overproduction of free radicals, whereas SOD is associated with free radical scavenging ability [19]. MDA also reflected the severity of lipid peroxidation, thus it often used as biomarker of oxidative stress [20].

In this study, the swimming training that were given to rats for certain duration and intensity produced a significant increase in oxidative stress biomarkers, principally shown through the elevation of MDA plasma and the decrease in SOD level $(p<0.05)$. MDA level was significantly found to be highest in exhaustive group (EX) than in control group (C) and moderate exercise group (M). The result was similar to other studies conducted in both animal and human studies, where exhaustive or intense exercise has been found to induce the elevation either in protein or lipid oxidation [21]. Yasa \& Jawi [22] found a sharp increase in MDA level after rats were forced to do treadmill exercise. From the previous studies we can see that exhaustive exercise enhanced lipid peroxidation that led to oxidative stress, resulting in dramatic increase in MDA level due to the rise of oxygen uptake during exercise [23]. The exhaustive exercise would cause blood flow to be redistributed, followed by ischaemia mechanism that in turn led to a serious reperfusion injury, as it has been shown by other previous studies [18]. Exhaustive exercise is also known to generate high level of plasma lactate, as an indicator of exercise intensity, which could also be contributed to free radicals production [23].

Although there are some debates regarding the source of free radical during exercise, mitochondria have been long considered as the major source of ROS [24]. In the mitochondria, the oxygen consumed partially loses one electron, giving rise to the superoxide radical $(\mathrm{O} 2 \bullet-)$, which is generated in different rates according to the examined tissue [25]. But as the intensity of exercise increased, the production of ROS mainly come from hypoxia condition [26]. During hypoxia, adenosine triphosphate (ATP) will decrease due to ATPdependent calcium ionic pump impairment and activation of $\mathrm{Ca}$-dependent proteases. This activity result in the cleavage of xanthine dehydrogenase to xanthine oxidase (XO). This enzyme catalyse a reaction where hypoxanthine were converted to xanthine then to uric acid and free radical $(\mathrm{O} 2 \bullet-)$ [18]. It explained why ROS production elevated sharply during exhaustive exercise. 
Regarding SOD level, we found that exhaustive group had the lowest SOD compared to the rest of the groups. The decrease in SOD levels that occurred in EX is caused by an imbalance between the amounts of antioxidants in the body and the number of free radicals formed due to exhaustive swimming training. This process causes oxidative stress, which is indicated by a decrease in SOD levels. The formation of malondialdehyde (MDA) will be followed by oxidative damage on the cell membrane, which in turn will lead to more progressive damage leading to decreased activity of intracellular enzymes such as Superoxide Dismutase (SOD), Glutathione Peroxide (GPx), and Catalase (CAT) [18].

Decrease of enzyme activity could also be a consequence of elated endogenous free radicals and MDA formation, as indicated by several studies. Previous study showed that, in response to oxidative stress, SOD enzyme might be consumed to prevent oxidative damage since it was shown that the overproduction of ROS exhausts the SOD capacity [27]. Apart from reducing enzyme activity, the high number of free radicals cause disturbance in the synthesis of antioxidant enzymes such as SOD and GPx. Disruption in enzyme synthesis will cause a decrease in the levels of SOD produced, so that the amount of SOD in the body cannot counterbalance the damage caused by free radicals that formed as a result of exhaustive exercise [28].

The oxidative stress happened to rats in moderate $(\mathrm{M})$ and exhaustive exercise (EX) indicated that swimming training could reduce SOD levels significantly. In addition, the sharp decrease in SOD levels in EX indicates that excessive exercise or physical activity does not provide a protective effect against free radicals. These results are in accordance with research conducted by Li et al [19], where giving excessive physical exercise can reduce the levels of SOD in Wistar rats lower than the control or baseline group.

\section{Conclusion}

Our findings indicate that exhaustive exercise gives significantly result in escalating malondialdehyde level and reducing the endogenous antioxidant levels, compared with control and moderate intensity exercise. Raised level of MDA and declined SOD are the potential risk factors for many diseases, so it is advised for people to do exercise in the right intensity and duration to maximize the health benefits. Further research is needed elucidate the long-term effects and potential mechanisms of high-intensity exercise and redox-mediated health adaptations.

\section{References}

[1] Elmagd MA. Benefits, need and importance of daily exercise. Int J Phys Educ Sport Heal [Internet]. 2016;3(5):22-7. Available from: www.kheljournal.com

[2] Li J, Siegrist J. Physical activity and risk of cardiovascular disease-a meta-analysis of prospective cohort studies. Int J Environ Res Public Health. 2012;9(2):391-407.

[3] Simioni C, Zauli G, Martelli AM, Vitale M, Sacchetti G, Gonelli A, et al. Oxidative stress: Role of physical exercise and antioxidant nutraceuticals in adulthood and aging. Oncotarget. 2018;9(24):17181-98.

[4] Brown JC, Winters-Stone L, Lee A, Schmitz KH. Cancer, physical activity, and exercise. Compr Physiol. 2014;2(4):2775-809.

[5] Klimek M, Knap J, Reda M, Masternak M. Physical activity in prevention and treatment of type 2 diabetes mellitus. J Educ Heal h Sport. 2019;9(11):1175-118. 
[6] Mandolesi L, Polverino A, Montuori S, Foti F, Ferraioli G, Sorrentino P, et al. Effects of physical exercise on cognitive functioning and wellbeing: Biological and psychological benefits. Front Psychol. 2018;9(April):1-11.

[7] Rodríguez-Fernández A, Zuazagoitia-Rey-Baltar A, Ramos-Díaz E. Quality of Life and Physical Activity: Their Relationship with Physical and Psychological Well-Being. In: Quality of Life and Quality of Working Life. 2017.

[8] Thirumalai T, Therasa SV, Elumalai EK, David E. Intense and exhaustive exercise induce oxidative stress in skeletal muscle. Asian Pacific J Trop Dis. 2011;1(1):63-6.

[9] Liao P, He Q, Zhou X, Ma K, Wen J, Chen H, et al. Repetitive bouts of exhaustive exercise induces a systemic inflammatory response and multi-organ damage in rats. Front Physiol. 2020;11(June):1-14.

[10] Mastorakos G, Pavlatou M. Exercise as a Stress Model and the Interplay Between the Hypothalamus-pituitary-adrenal and the Hypothalamus-pituitary-thyroid Axes. Horm Metab Res [Internet]. 2005;37(9):577-84. Available from: http://eprints.ncrm.ac.uk/2879/1/NCRM_workingpaper_0412.pdf

[11] Hoene M, Franken H, Fritsche L, Lehmann R, Pohl AK, Häring HU, et al. Activation of the mitogen-activated protein kinase (MAPK) signalling pathway in the liver of mice is related to plasma glucose levels after acute exercise. Diabetologia. 2010;53(6):1131-41.

[12] Al-Hashem FH, Shatoor AS, Sakr HF, Al-Daghri N, Khalil M, Alkhateeb M. Co-administration of vitamins $\mathrm{E}$ and $\mathrm{C}$ protects against stress-induceadhepatorenal oxidative damage and effectively improves lipid profile at both low and high altitude. African J Biotechnol. 2012;11(45):10416-23.

[13] Mittler R. ROS Are Good. Trends Plant Sci [Internet]. 2017 Jan;22(1):11-9. Available from: https://linkinghub.elsevier.com/retrieve/pii/S1360138516301121

[14] Pleńkowska J, Gabig-Cimińska M, Mozolewski P. Oxidative stress as an important contributor to the pathogenesis of psoriasis. Int J Mol Sci. 2020;21(17):1-15.

[15] Myint K, Jayakumar R, Hoe S, Kanthimathi MS, Lam SK. Cortisol, $\beta$-endorphin and oxidative stress markers in healthy medical students in response to examination stress. Biomed Res. 2017;28(8):3774-9.

[16] Sholikhah AM, Wirjatmadi B, Andriyani M. Effects of purple sweet potatoes on oxidative stress biomarkers in rats subjected to exhaustive exercise. Heal Notions. 2018;2(2):174-7.

[17] Parker L, Mcguckin TA, Leicht AS. Influence of exercise intensity on systemic oxidative stress and antioxidant capacity. Clin Physiol Funct Imaging. 2014;34(5):377-83.

[18] Kawamura T, Muraoka I. Exercise-induced oxidative stress and the effects of antioxidant intake from a physiological viewpoint. Antioxidants. 2018;7(9).

[19] Li XD, Sun GF, Zhu WB, Wang YH. Effects of high intensity exhaustive exercise on SOD, MDA, and NO levels in rats with knee osteoarthritis. Genet Mol Res. 2015;14(4):12367-76.

[20] Mulianto N. Malondialdehid sebagai Penanda Stres Oksidatif pada Berbagai Penyakit Kulit. Cermin Dunia Kedokt. 2020;47(1):42.

[21] Belviranli M, Gökbel H. Acute exercise induced oxidative stress and antioxidant changes. Eur J Gen Med. 2006;3(3):126-31.

[22] Yasa IWPS, Jawi IM. Antioxidant potential and hypolipidemic effects of combined purple sweet potato (Ipomoea batatas L.) tuber extract with honey in rats given high cholesterol feed. Bali Med J. 2017;6(3):65.

[23] Muñoz D, Olcina G, Timón R, Robles MC, Caballero MJ, Maynar M. Effect of different exercise intensities on oxidative stress markers and antioxidant response in trained cyclists. J Sports Med Phys Fitness. 2010;50(1):93-8.

[24] Khani M, Motamedi P, Dehkhoda MR, Dabagh Nikukheslat S, Karimi P. Effect of thyme extract supplementation on lipid peroxidation, antioxidant capacity, PGC-1 $\alpha$ content and endurance exercise performance in rats. J Int Soc Sports Nutr. 2017;14(1):1-8.

[25] Lima FD, Stamm DN, Della-Pace ID, Dobrachinski F, de Carvalho NR, Royes LFF, et al. Swimming training induces liver mitochondrial adaptations to oxidative stress in rats submitted to repeated exhaustive swimming bouts. PLoS One. 2013;8(2):1-9.

[26] Powers SK, Radak Z, Ji LL. Exercise-induced oxidative stress: past, present and future. J Physiol. 
2016;594(18):5081-92.

[27] Pejić S, Todorović A, Stojiljković V, Cvetković D, Lučić N, Radojičić RM, et al. Superoxide dismutase and lipid hydroperoxides in blood and endometrial tissue of patients with benign, hyperplastic and malignant endometrium. An Acad Bras Cienc. 2008;80(3):515-22.

[28] Poljsak B, Milisav I, Lampe T, Ostan I. Reproductive benefit of oxidative damage: An oxidative stress “malevolence"? Oxid Med Cell Longev. 2011;2011. 